\title{
Changes in Amplitude Characteristics of Heart Sound Signals during External-cuff-inflation Procedure: A Pilot Study
}

\author{
Xinpei Wang ${ }^{1}$, Yuanyang $\mathrm{Li}^{2}$, Chengyu Liu ${ }^{1}$, Changchun $\mathrm{Liu}^{1}$, Lizhen $\mathrm{Ji}^{1}$, Haibin Zeng ${ }^{1}$ \\ ${ }^{1}$ Institute of Biomedical Engineering, School of Control Science and Engineering, Shandong \\ University, Jinan, China \\ ${ }^{2}$ Shandong Provincial Hospital affiliated to Shandong University, Jinan, China
}

\begin{abstract}
Changes in amplitude characteristics of the first heart sound (S1) and second heart sound (S2) have been confirmed to be linked with the physiological or pathological changes of cardiovascular system. Earlier studies have also documented that these changes in amplitudes of S1 and S2 were correlated with blood pressure (BP) factor. External-cuff-inflation-deflation (ECID) is a standard procedure for BP measurement, and will certainly influence the BP state in the arterial trees. However, no existing studies report whether this ECID procedure will change the amplitude characteristics of S1 and S2. This study aimed to provide insight into this information.

Thirty-one healthy volunteers were enrolled. Heart sound and cuff pressure signals were recorded synchronously for each subject during a resting state and then followed by a external-cuff-inflation (ECI) state and $a$ external-cuff-deflation (ECD) state. The BP measurements included two patterns: cuff wrapped on the left upper arm and cuff wrapped on the two-side arms and legs. The amplitudes of S1 (S1_amp) and S2 (S2_amp), as well as their ratio (S1/S2), were calculated for each beat signals and then were averaged for each state. These three indices were compared between resting and ECI states, also between resting and ECD states using paired-t test. The results showed that S2_amp had significant differences between resting and ECI states with cuffs on the two-side arms and legs $(p<0.01)$.

To conclude, this pilot study suggested that the applied ECI procedure with cuffs on the two-side arms and legs during BP measurement significantly influences the amplitude characteristics of S2.
\end{abstract}

\section{Introduction}

Heart sound can provide valuable information associated with the valves and hemodynamics. The first heart sound (S1) relates to the closure of the mitral and the tricuspid valves and the second heart sound (S2) relates to the closure of the aortic and pulmonary valves. Heart sound has been used as a primary screening for various heart diseases [1].

Existing studies also have documented that the amplitudes of both S1 and S2 were associated with blood pressure (BP) factor. An existing study reported that amplitude of S2 from hypertensive patients was higher than that from normotensive [2]. Increased BP also led to an increase in frequency of S2 [3], [4]. The relation between S2 and the arterial BPs from both systemic and pulmonary circulations has been confirmed in [5], [6], [7]. In addition, the ratio of S1 to S2 displayed the high correlation with systolic BP values [8]. External-cuffinflation-deflation (ECID) is a standard procedure for BP measurement, and will certainly influence the BP state in the arterial trees. However, no existing studies report whether this ECID procedure will change the amplitude characteristics of S1 and S2. This study aimed to provide insight into this information.

\section{Method}

\subsection{Subjects}

Thirty-one subjects (12 male and 19 female; aged from 21 to 25 years) were studied. They were all healthy without any known diseases and gave their written informed consent. The detailed subject demographic information including age, height and weight were summarized in Table 1.

Table 1. Demographic data for the subjects studied. Their means and standard deviations (SDs) are presented.

\begin{tabular}{ll}
\hline Parameters & Values \\
\hline Number $(\mathrm{M} / \mathrm{F})$ & $31(12 / 19)$ \\
Age $($ year $)$ & $22 \pm 1$ \\
Height $(\mathrm{cm})$ & $169 \pm 7$ \\
Weight $(\mathrm{kg})$ & $57 \pm 9$ \\
\hline
\end{tabular}




\subsection{Protocol}

As shown in Figure 1, four cuffs were wrapped on two-side arms and legs to facilitate the cuff inflation and deflation procedure during $\mathrm{BP}$ measurement. A heart sound sensor was fixed at the heart position to obtain the heart sound during the cuff inflation and deflation. Thus phonocardiogram (PCG) and cuff pressure (CP) signals could be synchronously recorded by a Cardiovascular Function Detection device (CV FD-II, Huiyironggong Tech. Co., Ltd., Jinan, China) at a sample rate of $1000 \mathrm{~Hz}$. Subjects were required to lie flat and breathe regularly and gently during the measurements.

Firstly, only the cuff on the left upper arm was inflated and deflated. PCG and CP signals were recorded during the whole inflation and deflation procedure and subsequently were separated into three signal segments according to the measurement states: resting state, external-cuff-inflation (ECI) state and external-cuffdeflation (ECD) state. Thus we could compare the PCG characteristics among these three measurement sates.

Then, all four cuffs on the two-side arms and legs were synchronously inflated and deflated. PCG and CP signals were also recorded during the whole inflation and deflation procedure and subsequently were separated into three signal segments according to the measurement states: resting, ECI and ECD states.

During each BP measurement, the cuff was quickly inflated to block blood vessel under the cuff and then linearly deflated. Typical recorded signal waveforms during the aforementioned two process were shown in Figure 2.

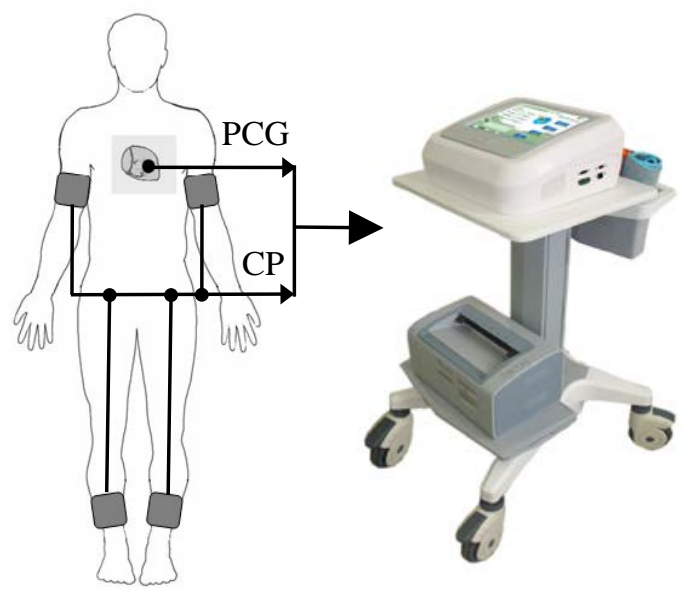

Figure 1. Diagram of the measurement process. PCG and $\mathrm{CP}$ signals were synchronously and digitally recorded using a Cardiovascular Function Detection device (CV FD-II).

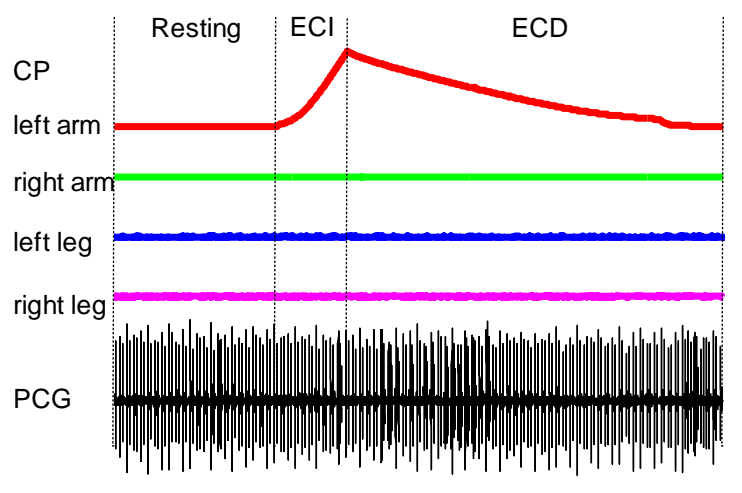

(a)

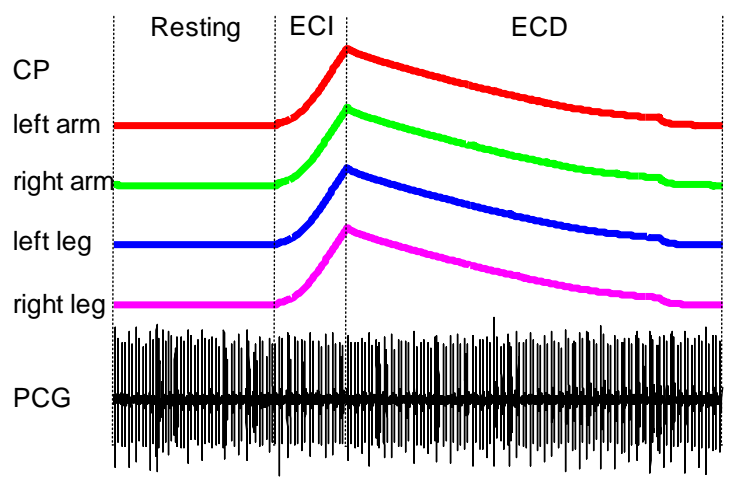

(b)

Figure 2. PCG and CP signals recorded under two BP measurement patterns: (a) only the cuff wrapped on the left upper arm was inflated and deflated, (b) all four cuffs wrapped on two-side arms and legs were synchronously inflated and deflated.

\subsection{Calculation of the indices}

S1 and S2 in PCG signal were identified automatically by a segmentation algorithm based on high-order Shannon entropy [9]. When the PCG signal was disturbed, the manual deletion was performed. An example of PCG signal with identified S1 and S2 was shown in Figure 3.

For each subject, PCG signals were firstly normalized. Then the mean amplitude values of S1, S2 and their ratio in each state were calculated as the heart sound indices (denoted as S1_amp, S2_amp and S1/S2 respectively).

\subsection{Statistical analysis}

Kolmogorov-Smirnov test was used to confirm the normal distributions of all heart sound indices. Paired $t$ test was used to compare of the indices differences between resting and ECI states, also between resting and 
ECD states respectively. All statistical analyses were performed using the SPSS software (Ver. 21, IBM, USA). A statistical significance was accepted at $\mathrm{P}<0.05$.

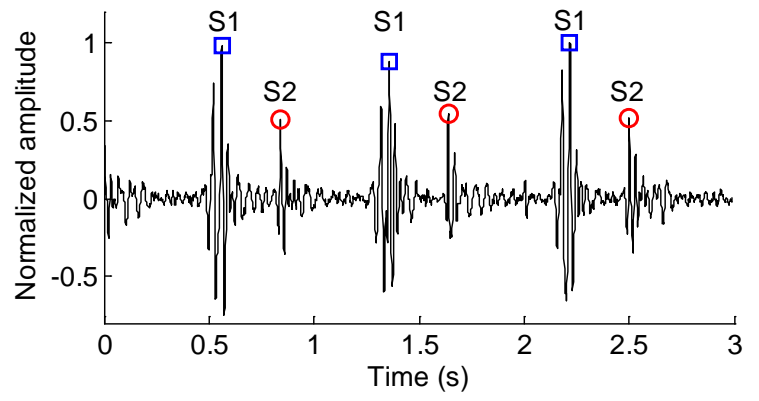

\section{Results}

The Kolmogorov-Smirnov test results confirmed that all heart sound indices were normal distributions ( $\mathrm{p}>0.05)$.

Table 2 showed the results of all heart sound indices under resting and ECI, ECD states. It demonstrated that there were no significant differences of all indices under resting and ECI, ECD states (p>0.05) except the S2_amp values under the resting and ECI states with cuffs on the two-side arms and legs. The S2_amp values under the resting state were significant lower than those under the ECI state with cuffs on the two-side arms and legs $(\mathrm{P}<0.01)$

Figure 3. An example of PCG signal with identified S1 and S2.

Table 2. Comparison of heart sound indices under resting and ECI, ECD states.

\begin{tabular}{llccccc}
\hline \multirow{2}{*}{ Cuffs } & Indices & \multicolumn{5}{c}{ Results under three states } \\
\cline { 3 - 7 } & & Resting & \multicolumn{2}{c}{ ECI } & \multicolumn{2}{c}{ ECD } \\
\cline { 3 - 6 } & & & Value & $P$ value & Value & $P$ value \\
\hline Only cuff on left arm & S1_amp & $0.76 \pm 0.18$ & $0.75 \pm 0.17$ & 0.5 & $0.76 \pm 0.17$ & 0.6 \\
& S2_amp & $0.57 \pm 0.17$ & $0.58 \pm 0.18$ & 0.2 & $0.58 \pm 0.17$ & 0.4 \\
& S1/S2 & $1.50 \pm 0.64$ & $1.46 \pm 0.64$ & 0.2 & $1.46 \pm 0.62$ & 0.2 \\
\hline All four cuffs & S1_amp & $0.76 \pm 0.18$ & $0.77 \pm 0.17$ & 0.3 & $0.75 \pm 0.19$ & 0.4 \\
& S2_amp & $0.59 \pm 0.16$ & $0.61 \pm 0.19$ & $<0.01$ & $0.59 \pm 0.18$ & 0.5 \\
& S1/S2 & $1.44 \pm 0.60$ & $1.41 \pm 0.60$ & 0.3 & $1.43 \pm 0.63$ & 0.8 \\
\hline
\end{tabular}

Note: data are shown in mean \pm standard deviation (SD). P value means the statistical results compared with the results under resting state.

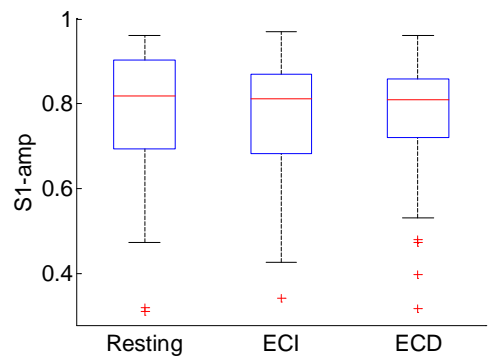

(a)

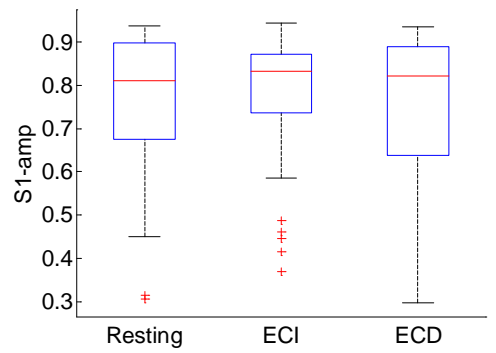

(d)

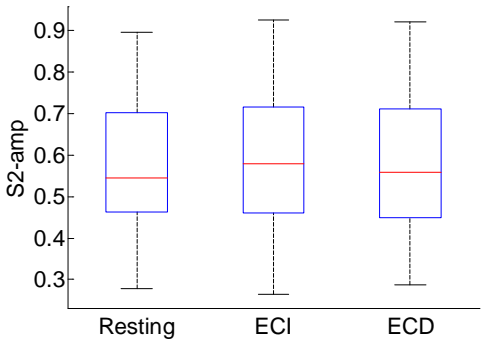

(b)

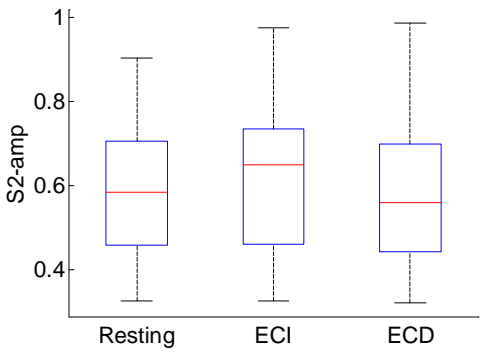

(e)

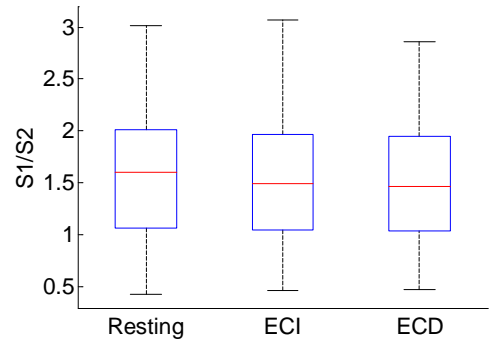

(c)

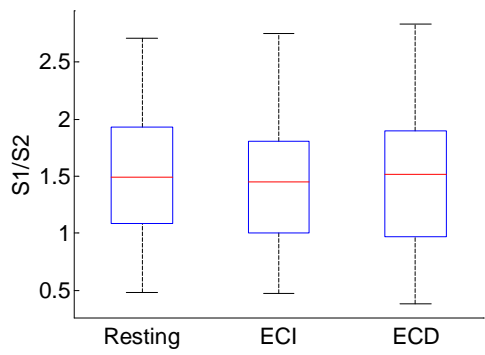

(f)

Figure 3. The boxplot graphs of the three heart sound indices under two BP measurement patterns: (a) S1_amp from the ECID with cuff on the left upper arm (ECID_lua), (b) S2_amp from the ECID_lua, (c) S1/S2 from the ECID_lua, (d) S1_amp from the ECID with cuffs on the two-side arms and legs (ECID_al), (e) S2_amp under the ECID_al and (f) S1/S2 under the ECID_al . 


\section{Discussion and conclusion}

It has been found in this study that the external-cuffinflation-deflation procedure on left arm during BP measurement hardly influenced the amplitude characteristics of S1 and S2. However, external-cuffinflation procedure on arms and legs synchronously significantly influenced the amplitude characteristics of S2. The external-cuff-inflation procedure on arms and legs synchronously led to an increase in the amplitude of S2. The results confirmed again the conclusion of the relation between S2 and BP reported by earlier studies. As reported, S2 is divided into A2 arising from the closure of aortic valve and P2 due to the closure of pulmonary valve, and the intensity of S2 increases with greater intensity in the closure of aortic valve. The internal pressure of the aorta is the force that closes the aortic valve [8]. Thus, the increase of the S2 amplitudes is due to the increase of the aortic pressure。

This pilot study demonstrated that the external-cuffinflation-deflation procedure on left upper arm had no significantly effect on the aortic pressure. However, the external-cuff-inflation procedure on both arms and legs synchronously is a beneficial attempt to change the aortic pressure.

\section{Acknowledgements}

This work was supported by the National Natural Science Foundation of China (No. 61501280, 61471223, 61671275), the Excellent Young Scientist Awarded Foundation of Shandong Province (No. BS2012DX019) and Shandong Provincial Science and Technology Project (No. 2014GSF118030, 2015GSF118179).

\section{References}

[1] Durand LG, Pilbarot P. Digital signal processing of the phonocardiogram: review of the most recent advancements. Critical Reviews in Biomedical Engineering. 1995; 23: 163-
219.

[2] Bartels A, Harder D. Non-invasive determination of systolic blood pressure by heart sound pattern analysis. Clin. Phys, Physiol. Meas.. 1992; 13: 249-256.

[3] Arnott P, Pfeiffer G, Tavel M. Spectral analysis of heart sounds: Relationships between some physical characteristics and frequency spectra of first and second heart sounds in normals and hypertensives. Journal of Biomedical Engineering. 1984; 6(2): 121-128.

[4] Peng RC, Yan WR, Zhang NL, Lin WH, Zhou XL, YuanTing Zhang. Cuffless and Continuous Blood Pressure Estimation from the Heart Sound Signals. Sensors. 2015; 15: 23653-23666.

[5] Bartels A, Harder D. Non-invasive determination of systolic blood pressure by heart sound pattern analysis. Clinical Physics and Physiological Measurement. 1992; 13(3): 249256.

[6] Xu J, Durand LG, Pibarot P. Extraction of the aortic and pulmonary components of the second heart sound using a nonlinear transient chirp signal model. IEEE Transactions on Biomedical Engineering. 2001; 48(3): 277-283.

[7] Smith R, Ventura D. A general model for continuous noninvasive pulmonary artery pressure estimation. Computers in Biology and Medicine. 2013; 43(7): 904-913.

[8] Lim KH, Shin YD, Park SH, Bae JH, Lee HJ, Kim SJ, Shin JY, Choi YJ. Correlation of blood pressure and the ratio of S1 to S2 as measured by esophageal stethoscope and wireless bluetooth transmission. Pak J Med Sci. 2013; 29: 1023-1027.

[9] Wang XP, Li YY, Sun CR, Liu CC. Detection of the First and Second Heart Sound Using Heart Sound Energy. $2^{\text {nd }}$ International Conference on Biomedical Engineering and Information, 2009: 478-481.

Address for correspondence.

Xinpei Wang

Institute of Biomedical Engineering

School of Control Science and Engineering

Shandong University

No.17923, Jingshi Road, Jinan, China, 250061

E-mail: wangxinpei@sdu.edu.cn 